\title{
ESTUDIO DE LOS MÉTODOS ANALÍTICOS PARA LA EXTRACCIÓN DE PARÁMETROS ELÉCTRICOS DE MÓDULOS FOTOVOLTAICOS DE CAPAS DELGADAS
}

\author{
STUDY OF ANALYTICAL METHODS FOR EXTRACTING ELECTRIC \\ PARAMETERS OF THIN-FILM PHOTOVOLTAIC MODULES
}
Renzo Perich- Ibáñez ${ }^{1}$, Miguel Sevillano-Bendezú' ${ }^{1}$, Jesús Montes-Romero' ${ }^{2}$, Luis Conde-Mendoza ${ }^{1}$, José Angulo-Abanto ${ }^{1}{ }^{(}$, Juan de la Casa-Higueras ${ }^{2}{ }^{\mathbb{C}}$, Jan Palomino-Töfflinger ${ }^{{ }^{*}}$ (b)

'Departamento de Ciencias, Sección Física, Pontificia Universidad Católica del Perú, Lima, Perú

${ }^{2}$ Grupo IDEA, Departamento de Ingeniería Electrónica y Automatización, Universidad de Jaén, Jaén, España

Recibido (Recieved): 21/02/2020 Aceptado (Accepted): 05/03/2020

\section{RESUMEN}

Para conocer la eficiencia real de un panel y poder predecir su potencia y producción energética es necesario estudiar su comportamiento y caracterizarlo a las condiciones reales del lugar en el que está instalado. El modelo de un solo diodo para celdas fotovoltaicas (FV) relaciona la corriente y el voltaje del módulo FV mediante cinco parámetros eléctricos que nos dan información fundamental acerca de los procesos físicos que tienen lugar en las celdas FV y del estado del módulo. Actualmente, existen diversos métodos analíticos, numéricos y heurísticos para extraer los parámetros del modelo, cada uno con ventajas y desventajas que dependen del tipo de módulo y de las condiciones ambientales. Se realizó un estudio de métodos de extracción analíticos que se usan actualmente para módulos FV de silicio cristalino y se aplicaron a tecnologías FV de capas delgadas. En este trabajo se presentan los resultados de la extracción de parámetros a través de comparación entre la curva corriente-voltaje (IV) medida y la modelada, además de cálculos de Normalized Root Mean Square Error (NRMSE) con el fin de comparar y evaluar ambos métodos analíticos.

Palabras Clave: fotovoltaica, modelado de tecnologías de lámina delgada, modelo de un solo diodo, extracción de parámetros, curva IV

\section{ABSTRACT}

In order to find a module's real efficiency and predict its power and energy production it is necessary to study its behaviour and characterize it in the conditions in which it is installed. The single diode model for photovoltaic (PV) cells relates a cell's current to its voltage through five electric parameters which give us fundamental information about a module and the physical processes inside its cells. Currently, there are many analytical, numerical and heuristic methods used to extract the model's parameters, each one with advantages and disadvantages that depend on the technology of the module and climate conditions. Current analytical methods used to extract parameters from silicon modules were applied to thin film PV modules in order to study their validity. This work presents the results of the parameter extraction by comparing the measured current-voltage (IV) curve with the modelled curve, as well as through Normalized Root Mean Square Error (NRMSE) calculations with the objective of comparing and evaluating the selected analytical methods.

Keywords: photovoltaics, modelling thin film technologies, single diode model, parameter extraction, IV curve

\footnotetext{
${ }^{1 *}$ Corresponding author.:

E-mail: japalominot@pucp.edu.pe 


\section{INTRODUCCIÓN}

Al no emitir gases contaminantes durante su operación, ofrecer la posibilidad de generar energía cerca o dentro de ciudades y poseer una fuente inagotable de energía, los paneles fotovoltaicos se han convertido en una de las mejores alternativas para generar energía limpia. Actualmente el mercado FV es dominado por las tecnologías de silicio cristalino [1]. Las celdas FV de película delgada poseen algunas ventajas sobre las celdas de silicio cristalino: menor costo debido a que se utiliza menos material semiconductor y la posibilidad de crear celdas flexibles [2]. Debido a esto hay motivo por el cual dedicar mayor atención al estudio de estas tecnologías. Para demostrar que estas tecnologías realmente tienen la capacidad de competir con las tecnologías de silicio, se necesitan proyectos que estudien el comportamiento de las celdas una vez que hayan sido instaladas.

Esta investigación se enfocó en la caracterización a condiciones reales de módulos FV de películas delgadas. La caracterización permite comprender mejor el funcionamiento de las tecnologías FV y así conocer más acerca de sus ventajas y desventajas para saber en qué condiciones resulta más conveniente utilizar cada tecnología. Actualmente los fabricantes incluyen información acerca de las propiedades eléctricas de cada panel, pero estas no son suficientes para evaluar su calidad. Adicionalmente se sabe que la información incluida por el fabricante es válida para condiciones estándar (STC) [3] y en la práctica los módulos van a estar expuestos a distintas combinaciones de irradiancia y temperatura.

\section{METODOLOGÍA}

La caracterización de los módulos estudiados se realizó mediante el análisis de curvas de corrientevoltaje (IV) y el uso del modelo de un solo diodo. Este representa una celda $F V$ usando un generador de corriente conectado en paralelo con un diodo, ambos conectados en paralelo con una carga. Finalmente incluye un par de resistencias, en serie y en paralelo para representar pérdidas eléctricas.

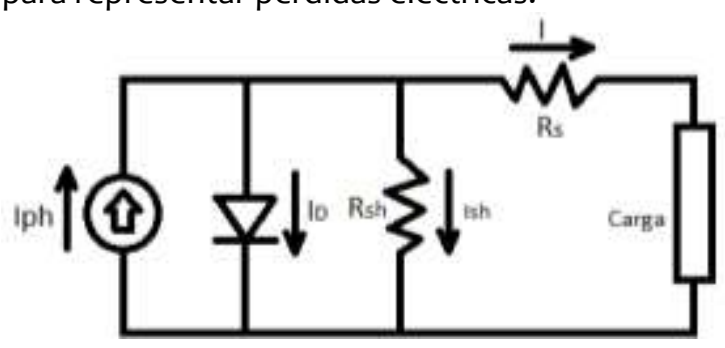

Fig. 1. Circuito equivalente de una celda en el modelo de un solo diodo.

Utilizando leyes de Kirchhoff se le puede restar a la fotocorriente la corriente del diodo [ec. (1)] y la corriente fuga de la resistencia en paralelo [ec. (2)]. De esta forma se obtiene una ecuación que relaciona la corriente y voltaje en la celda FV [ec. (4)].

$$
\begin{gathered}
I_{D}=I_{0} \exp \left(\frac{V+I R_{s}}{n V_{T}}\right) \\
I_{s h}=\frac{V+I R_{s}}{R_{s h}} \\
I=I_{p h}-I_{D}-I_{s h} \\
I=I_{p h}-I_{0} \exp \left(\frac{V+I R_{s}}{n V_{T}}\right)-\frac{V+I R_{s}}{R_{s h}}
\end{gathered}
$$

En la ecuación del modelo de un solo diodo aparecen cinco parámetros eléctricos que tienen un significado físico asociado que proporciona información valiosa acerca de la calidad de la celda. La fotocorriente $\left(I_{p h}\right)$ representa la corriente total generada por el efecto fotovoltaico que se da en la celda. La resistencia en serie $\left(R_{s}\right)$ se incluye para tomar en cuenta las pérdidas eléctricas debido a la resistencia de los contactos mientras que la resistencia en paralelo $\left(R_{s h}\right)$ toma en cuenta las pérdidas por corrientes fuga [4]. El factor de idealidad del diodo (n) contiene información acerca de en qué parte de la celda se están dando las recombinaciones. Finalmente, la corriente de saturación del diodo $\left(I_{0}\right)$ representa la difusión y recombinación de cargas en la unión PN [2].

El modelo de un solo diodo fue escogido por su simplicidad y habilidad para recrear la curva IV de manera exitosa [2]. Existen modelos más complejos como el modelo de dos diodos, pero este introduce dos parámetros nuevos (un nuevo par de lo y $n$ ) a la ecuación complicando su solución. Además, las ventajas del segundo diodo están relacionadas una mejor aproximación de la corriente a oscuras [4] y en un estudio de módulos instalados a condiciones reales se espera alcanzar irradiancias muy por encima de lo que podría considerarse condiciones oscuras.

Se estudiaron dos métodos analíticos que permiten extraer estos cinco parámetros característicos de curvas IV experimentales. La ventaja de los métodos analíticos sobre los métodos numéricos está en que requieren menor esfuerzo computacional y son más simples de implementar. Además, los métodos numéricos necesitan de valores iniciales para comenzar a iterar, si estos no están lo suficientemente cerca al valor real el método puede no converger. Los métodos escogidos fueron formulados para ser utilizados en tecnologías de silicio cristalino tradicional, en este estudio se han aplicado a tecnologías de segunda generación para evaluar su validez. 
En este análisis se trabajó con un set experimental de curvas características de módulos FV, que fueron obtenidas de simultáneamente con la temperatura del módulo y resto de parámetros ambientales en los laboratorios de la Universidad de Jaén (España). Además, se utilizaron datos de módulos de silicio cristalino que fueron instalados en la Pontificia Universidad Católica del Perú para comparar los resultados obtenidos.

Se escogieron curvas IV características en base a la irradiancia y temperatura ambiental. Para este estudio se trabajó con datos que fueron tomados cerca de $1000 \mathrm{~W} / \mathrm{m}^{2}$ y $25{ }^{\circ} \mathrm{C}$ ambiental, con temperaturas de módulos entre $50.0^{\circ} \mathrm{C}$ y $57.0^{\circ} \mathrm{C}$.

Las tecnologías de película delgada bajo estudio fueron:

- $\quad$ Hydrogenated amorphous silicon (a-Si:H)

- $\quad$ Copper Indium Selenide (CIS)

- $\quad$ Cadmium Telluride (CdTe)

- Tandem de amorphous silicon/micro-

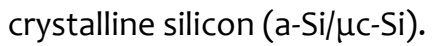

Tecnología de silicio policristalino (control):

- $\quad$ Aluminium Back Surface Field (AL-BSF)

Existen múltiples métodos de extracción de parámetros, por la afinidad del grupo de la PUCP con el grupo IDEA de la Universidad de Jaén, esta investigación se centró en los métodos analíticos de Khan et al. [5] y Phang et al. [6] que fueron investigados por Montes-Romero et al. [7] y Fernández et al. [8].

Ambos métodos fueron formulados para extraer los parámetros eléctricos de módulos de silicio policristalino utilizando puntos característicos de la curva IV experimental. Estos son la corriente cortocircuito $\left(I_{s c}\right)$, el voltaje a circuito abierto $\left(V_{o c}\right)$, el voltaje en el punto de máxima potencia $\left(V_{m p p}\right)$ y la corriente en el punto de máxima potencia ( $I_{m p p}$ ). Además de estos puntos, se utiliza el valor de las pendientes de la curva $I V$ cerca de la $I_{s c}$ y el $V_{o c}$.

Estas se obtienen imponiendo un fit lineal a los datos de 0 a $30 \%$ de la $I_{\text {sc }}$ para la pendiente en el $V_{\text {oc }} y$ otro fit a los datos de 0 a $30 \%$ del $V_{\text {oc }}$ para la pendiente de la $I_{s c}$ (fig. 2). En los casos en los que la data no estuvo completa a los extremos, el fit lineal se hizo usando los valores máximos de $\mathrm{V}$ e I en lugar de el $\mathrm{V}_{o c} \mathrm{e}$ $\mathrm{I}_{\mathrm{sc}}$. Se utilizaron las rectas para extrapolar y obtener el $V_{o c}$ e $I_{s c}$.

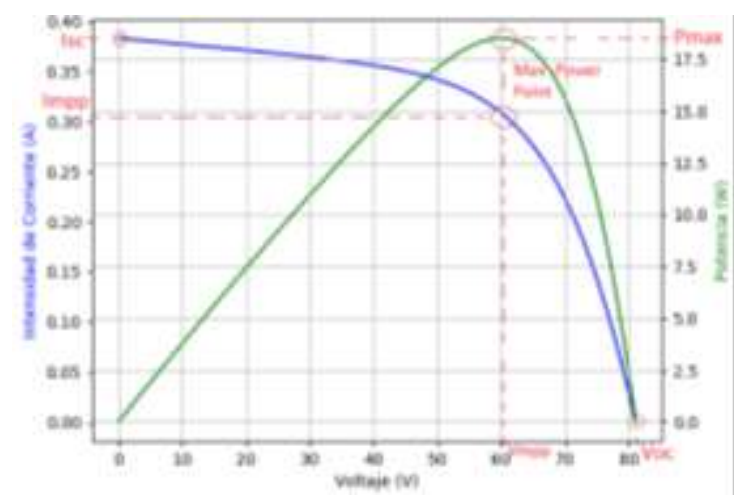

(a)

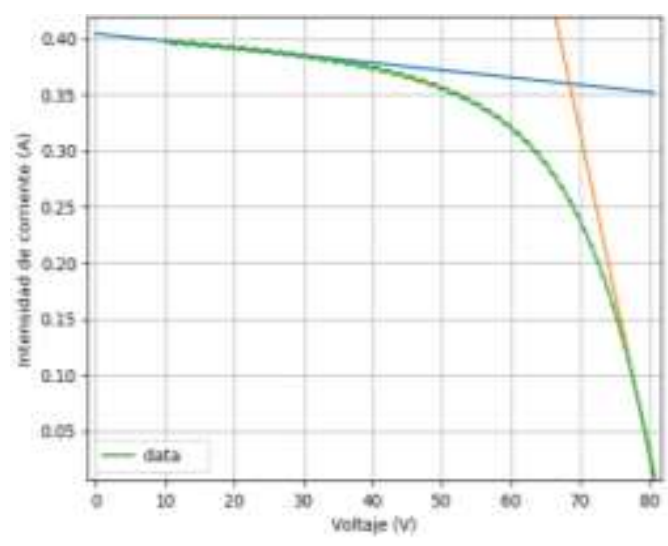

(b)

Fig. 2. (a) Curva IV y potencia-voltaje experimental mostrando los puntos característicos. (b) Curva IV experimental con fit lineal en en la región del $V_{\text {oc }}$ y la $I_{s c}$.

La inversa de las pendientes se utiliza para estimar las resistencias $R_{s}$ y $R_{s h}$ usando las ecuaciones (5) y (6).

$R_{s o}=-\left.\frac{d V}{d I}\right|_{v}={ }_{v_{o c}}$

$R_{\text {sho }}=-\left.\frac{d V}{d I}\right|_{I}={ }_{I_{S c}}$

Los puntos característicos de la curva IV junto con las resistencias estimadas Rso y Rsho se pueden introducir en la ecuación (4) para generar un sistema de cinco ecuaciones con los cinco parámetros como incógnitas. Estas ecuaciones se resuelven introduciendo las suposiciones sugeridas por cada autor para simplificar el sistema y desacoplarlo.

\subsection{MÉTODO DE PHANG ET AL.}

Este método analítico ofrece cinco ecuaciones que se pueden ordenar para ser resueltas usando los puntos característicos de la curva, Rso, Rsho y parámetros del modelo que ya han sido calculados.

$$
R_{\text {sh }}=R_{\text {sho }}
$$

$n=$ 


$$
\begin{gathered}
\frac{V_{m p p}+R_{s o} I_{m p p}-V_{o c}}{\left.V_{T}\left(\ln \left(I_{s c}-\frac{V_{m p p}}{R_{s h}}-I_{m p p}\right)-\ln \left(I_{s c}-\frac{V_{o c}}{R_{s h}}\right)\right)+\frac{I_{m p p}}{I_{s c}-\frac{V_{o c}}{R_{S h}}}\right)} \\
I_{0}=\left(I_{s c}-\frac{V_{o c}}{R_{s h}}\right) \exp \left(\frac{-V_{o c}}{n V_{T}}\right) \\
R_{s}=R_{s o}-\frac{n V_{T}}{I_{0}} \exp \left(\frac{-V_{o c}}{n V_{T}}\right) \\
I_{p h}=I_{s c}\left(1+\frac{R_{s}}{R_{s h}}\right)+I_{0}\left(\exp \left(\frac{I_{s c} R_{s}}{n V_{T}}\right)-1\right)
\end{gathered}
$$

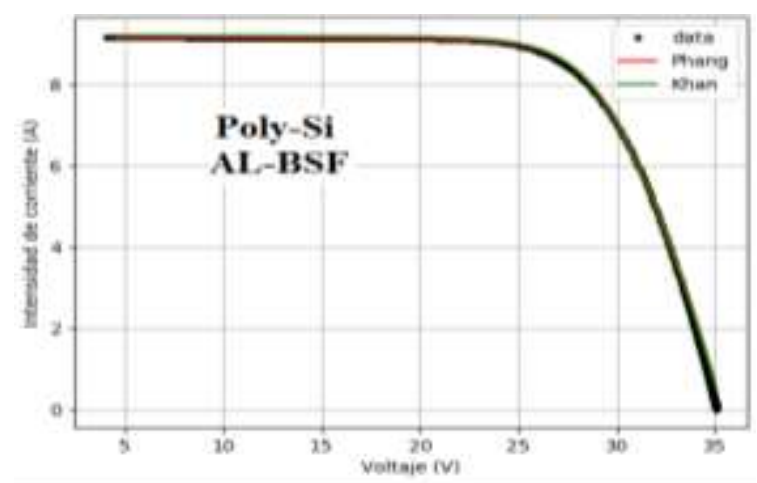

(a)

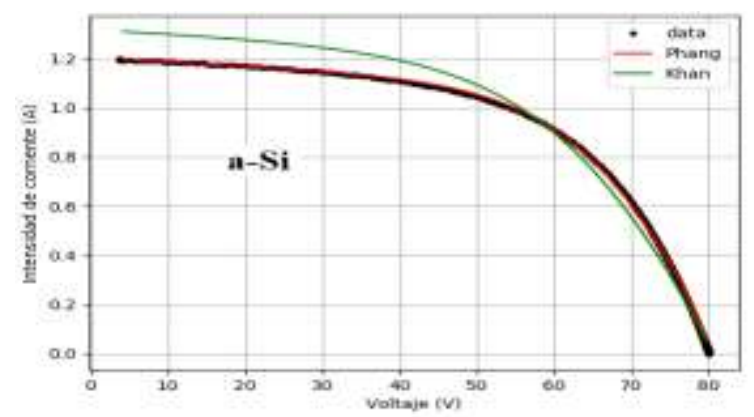

(b)

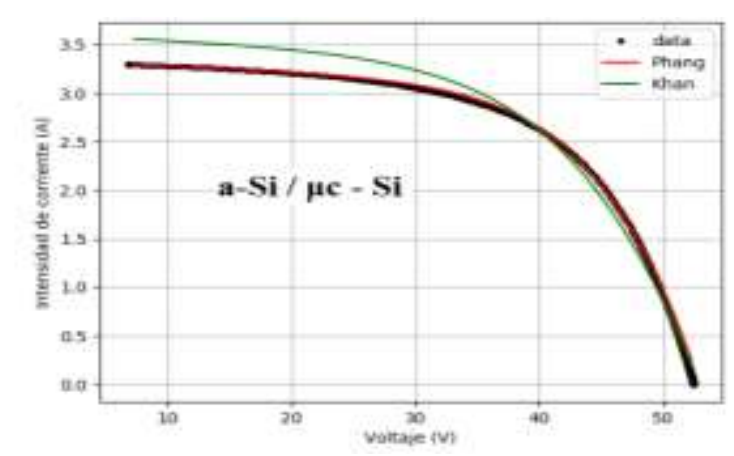

(c)

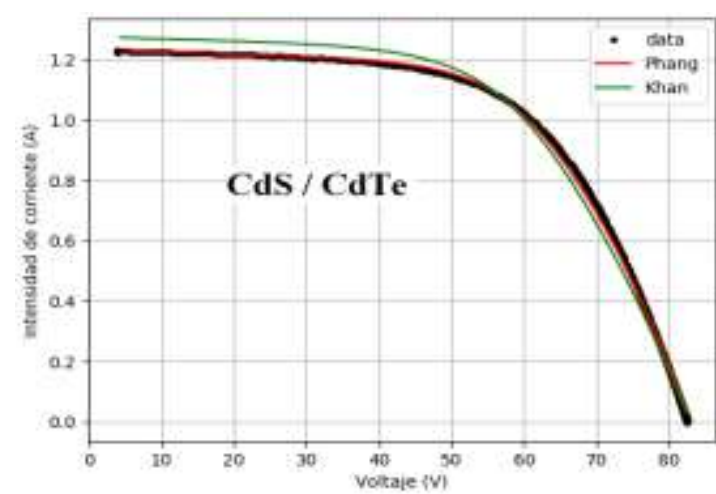

(d)

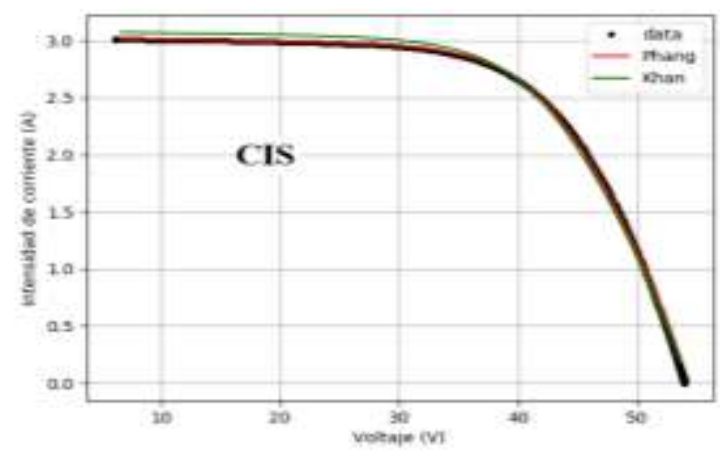

(e)

Fig. 3 Comparación entre la curva IV medida y las modeladas usando los métodos de Phang y Khan. (a) Silicio Policristalino: Aluminium Back Surface Field, (b) Silicio amorfo, (c) Tandem de Silicio Amorfo y Silicio Microcristalino, (d) Telururo de Cadmio y (e) Copper Indium Selenide.

\subsection{MÉTODO DE KHAN ET AL.}

En este método se utiliza la ecuación (7) del método de Phang para calcular la resistencia en paralelo. Luego presenta nuevas ecuaciones que permiten calcular los parámetros restantes.

$$
\begin{gathered}
R_{s}=R_{s o}-\frac{V_{m p p}+R_{s o} I_{m p p}-V_{o c}}{I_{m p p}+\left(\ln \left(I_{s c}-I_{m p p}\right)-\ln \left(I_{s c}\right)\right) I_{s c}} \\
I_{0}=\frac{n V_{T}}{\left(R_{s o}-R_{s}\right)} \exp \left(\frac{-V_{o c}}{n V_{T}}\right) \\
n=\frac{V_{m p p}+R_{s} I_{m p p}-V_{o c}}{V_{T}\left(\ln \left(I_{s c}-I_{m p p}\right)-\ln \left(I_{s c}\right)\right)} \\
I_{p h}=I_{0}\left(\exp \left(\frac{V_{o c}}{n V_{T}}\right)-1\right)+\frac{V_{o c}}{R_{s h}}
\end{gathered}
$$

Habiendo obtenido los parámetros del modelo de un solo diodo con cada método, se utilizaron para recrear la curva IV experimental. El error de la extracción se evaluó mediante comparación entre la curva IV experimental y la modelada usando la ecuación (4) y los parámetros extraídos. Se compararon usando el parámetro Normalized Root Mean Square Error (NRMSE). 
NRMSE $=\sqrt{-\sum_{N} \sum_{i=1}\left(\frac{I_{\text {modelado }}(V)_{i}-I_{\text {medido }}(V)_{i}}{I_{\text {sc, medido }}}\right)^{2}}$

Donde $\mathrm{N}$ es el número total de puntos utilizados para calcular el error, $I_{\text {modelada }}$ es la corriente calculada usando el modelo de un solo diodo e $I_{\text {medida }}$ es la corriente que se obtuvo en el trazado experimental de la curva IV. Finalmente se divide por el valor medido de la fotocorriente $\left(I_{s c, \text { medido }}\right)$ para normalizar el error.

\section{ANÁLISIS DE RESULTADOS}

Ambos métodos permitieron reproducir las curvas IV experimentales para todas las tecnologías, con diferentes grados de error. La fig. 3 (a) muestra la curva IV de un módulo de silicio policristalino instalado en la Pontificia Universidad Católica del Perú, además de las curvas modeladas usando los métodos de Phang y Khan. Se puede verificar que los métodos son capaces de reproducir la curva IV de un módulo de silicio.

Las figuras 3 (b), (c), (d) y (e) muestran los resultados de la extracción de parámetros para los módulos de película delgada instalados en la Universidad de Jaén.

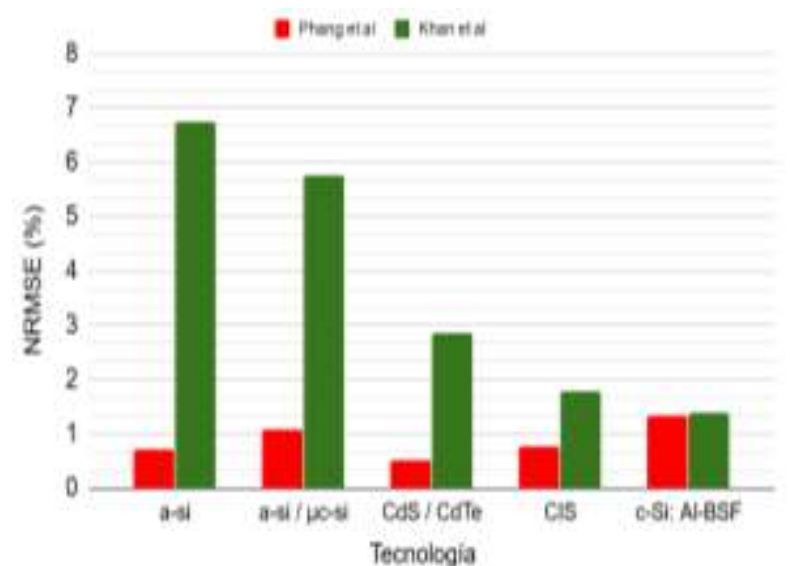

Fig. 4 Comparación del error entre la curva IV medida y la modelada, para cada método y tecnología

La gráfica del error (Fig. 4) para cada tecnología muestra como el método de Khan obtiene un error muy distinto para cada tecnología. El método propuesto por Phang obtiene un error que oscila cerca del $1 \%$.

\section{CONCLUSIONES}

- Está demostrado que los métodos de Phang et al. [6] y Khan et al. [5] son efectivos para extraer los parámetros asociados al modelo de un solo diodo en módulos fotovoltaicos cuyo material absorbente es el silicio policristalino (c-Si). Los módulos de c-Si tienen curvas IV más cercanas a la de un diodo ideal (Rs $\rightarrow 0$, Rsh $\rightarrow$ infinito) con una pendiente más cercana a cero y menos curvatura en la vecindad de la Isc. Esto permite hacer un ajuste lineal en los extremos para obtener las derivadas en el Voc e Isc con precisión.

- Al observar las curvas IV medidas para las tecnologías de película delgada se puede ver que las curvas tienen pendientes más inclinadas y menos constantes en la proximidad de la Isc. Esto introduce error a la hora hacer el fit lineal y afecta la extrapolación para obtener la Isc y el Voc.

- De la figura 4 se evidencia que el NRMSE de la curva simulada basada en los parámetros extraídos según el método de Phang es menor para todas las tecnologías estudiadas. El sistema de ecuaciones para el método de Phang sugiere una aproximación para la Iph que es proporcional a la Isc y a términos que son pequeños en comparación a Isc (ec. 11). Esto asegura que la curva simulada con los parámetros de Phang se aproxime correctamente a la curva medida en la vecindad del Isc. Un mayor e inestable NRMSE para el método de Khan podría deberse a que la expresión que proporciona para el Iph (ec. 14) no está directamente relacionada a la Isc.

- Así, el NRMSE permitió evaluar el ajuste de las curvas I-V simuladas en base a los métodos de Phang y Khan para cuatro diferentes módulos de películas delgadas, reportándose de estas una mejor aproximación con el método de Phang, aunque aún se requieren mayores estudios a fin de entender los parámetros asociados al modelo de un solo diodo aplicado a módulos de películas delgadas para los métodos analíticos presentados.

\section{AGRADECIMIENTOS}

Agradecimientos al grupo IDEA de la Universidad de Jaén. Este trabajo recibió apoyo financiero por el Fondo Nacional de Desarrollo Científico, Tecnológico y de Innovación Tecnológica (FONDECYT) mediante el proyecto con el contrato $\mathrm{N}^{\circ} 124-2018-F O N D E C Y T$.

\section{REFERENCIAS}

[1] G. Ciulla, V. Lo Brano, V. Di Dio and G. Cipriani. "A comparison of different one-diode models for the representation of I-V characteristic of a PV cell", Renewable and Sustainable Energy Reviews, vol.32, pp. 684-696, 2014. 
[2] M. Kumar and A. Kumar, "Performance assessment and degradation analysis of solar photovoltaic technologies: A review", Renewable and Sustainable Energy Reviews, vol. 78, pp. 554-587, 2017.

[3] F. Ghani, G. Rosengarten, M. Duke and J. K. Carson, "The numerical calculation of single-diode solar-cell modelling parameters", Renewable Energy, vol. 72, pp. 105-112, 2014.

[4] A. R. Jordehi, "Parameter estimation of solar photovoltaic (PV) cells: A review", Renewable and Sustainable Energy Reviews, vol.61, pp. 354-371, 2016.

[5] F. Khan, S.-H. Baek, Y. Park and J. H. Kim, "Extraction of diode parameters of silicon solar cells under high illumination conditions", Energy Conversion and Management, vol. 76, pp. 421-429. 2013.

[6] J. C. H. Phang, D. S. H. Chan and J. R. Phillips, "Accurate analytical method for the extraction of solar cell model parameters", Electronics Letters, vol. 20, no. 10, pp. 406-408, 1984.

[7] J. Montes-Romero, F. Almonacid, M. Theristis, J. de la Casa, G. E. Georghiou, and E. F. Fernández, "Comparative analysis of parameter extraction techniques for the electrical characterization of multijunction CPV and m-Si technologies", Solar Energy, vol.160, pp. 275-288, 2018.

[8] E. F. Fernández, J. Montes-Romero, J. de la Casa, PRodrigo F. and Almonacid, "Comparative study of methods for the extraction of concentrator photovoltaic module parameters", Solar Energy, vol. 137, pp. 413-423, 2016. 